\title{
Flaubert. Éthique et Esthétique, sous la direction d'Anne Herschberg Pierrot
}

\section{Maria Emanuela Raffi}

\section{(2) OpenEdition}

1 Journals

\section{Edizione digitale}

URL: http://journals.openedition.org/studifrancesi/3808

DOI: 10.4000/studifrancesi.3808

ISSN: 2421-5856

\section{Editore}

Rosenberg \& Sellier

\section{Edizione cartacea}

Data di pubblicazione: 1 décembre 2012

Paginazione: 595-596

ISSN: 0039-2944

\section{Notizia bibliografica digitale}

Maria Emanuela Raffi, «Flaubert. Éthique et Esthétique, sous la direction d'Anne Herschberg Pierrot», Studi Francesi [Online], 168 (LVI | III) | 2012, online dal 30 novembre 2015, consultato il 05 mars 2021. URL: http://journals.openedition.org/studifrancesi/3808; DOI: https://doi.org/10.4000/studifrancesi. 3808

Questo documento è stato generato automaticamente il 5 mars 2021.

\section{(c) $(1) \ominus$}

Studi Francesi è distribuita con Licenza Creative Commons Attribuzione - Non commerciale - Non opere derivate 4.0 Internazionale. 


\title{
Flaubert. Éthique et Esthétique, sous la direction d'Anne Herschberg Pierrot
}

\author{
Maria Emanuela Raffi
}

\section{NOTIZIA}

AA. VV., Flaubert. Éthique et Esthétique, sous la direction d'Anne HERSCHBERG PIERROT, Presses Universitaires de Vincennes, Paris, 2012, pp. 236.

1 La vera etica di Flaubert, sostiene la curatrice del volume nell'Avant-propos, sta nell'estetica dell'impersonalità, che «refuse de transformer l'œuvre en réceptacle de l'opinion de son auteur». La coincidenza del vero, del buono e del bello, unici valori esistenti agli occhi di Flaubert in quanto valori dell'opera e non dell'autore, consacra l'autonomia dell'arte sottraendola a qualunque finalità utilitaristica e valorizza la scrittura come «absolu de l'œuvre».

2 Pierre BERGOUNIOUX, con La littérature comme lutte à mort des consciences, parte da alcune precisazioni sulla nozione di «réalisme», da Balzac a Stendhal e infine a Flaubert, rilevando che i romanzi flaubertiani più noti, Madame Bovary e L'Éducation sentimentale prima di tutti, ingaggiano "une lutte symbolique à mort contre le monde» che descrivono, avendo come scopo quello di vuotare di senso l'esistenza della classe sociale di cui colpiscono l'indifferenza e la degradazione, senza tuttavia poter garantire del tutto l'agognata estraneità dell'autore, la sua completa «mort au monde».

3 Puissance de l'infime di Jacques RANCIÈRE affronta le critiche mosse a Flaubert per il carattere minuzioso delle sue descrizioni. L'estetica dell'infimo, che divide in atomi indifferenziati la realtà, viene invece per Rancière da un'etica specifica, che passa per «l'abolition de tout jugement», e da una concezione dell'arte intesa come "travail d'esténuation de la matière solide». Dal confronto con due opere contemporanee di Ruskin e Whitman, che come Madame Bovary riflettono sul legame fra etica ed estetica in modi peraltro assai diversi -, l'A. definisce una sorta di «démocratisme littéraire», comune a Flaubert e allo scrittore statunitense. Tuttavia, se entrambi definiscono 
un'idea di poesia impersonale che dia voce al pensiero e alla vita presenti in ogni minuscola cosa, Flaubert si separa da Whitman, poiché per l'autore di Madame Bovary i piccoli avvenimenti sensibili funzionano come «fenêtres ouvertes sur l'infini» non per i personaggi, ma solo nella scrittura, solo nel libro.

4 Agnès BOUvIER affronta in Éthique et esthétique de la traduction dans "Salammbô" il problema della traduzione come adeguamento della lingua della narrazione alla situazione linguistica e storico-culturale rappresentata. Per arrivare alla lingua punica attraverso l'ebraico, Flaubert si vede costretto a prendere come testo di riferimento la Bibbia di Cahen, prima traduzione "moderna" della Bibbia, tratta direttamente dall'ebraico senza la mediazione della Vulgata. Il lavoro di traduzione e di annotazione del lessico derivato dall'ebraico, che la Bouvier presenta attraverso numerosi esempi inclusa la parola "punica" inventata da Flaubert: zaïmph -, mostra il considerevole impegno dello scrittore per preservare il carattere singolare della lingua di partenza, l'ebraico, in un periodo in cui la pratica delle «belles infidèles» era assai più gradita della fedeltà letterale.

5 «Emma Bovary et le bovarysme», seconda sezione del volume, si apre con Qui a tué Madame Bovary?, in cui Françoise GALLARD prende spunto dal romanzo poliziesco di Philippe Doumenc, Contre-enquête sur la mort d'Emma Bovary (2007) per indagare le ragioni della condanna a morte dell'eroina da parte di Flaubert, colpevole ai suoi occhi non solo di aver letto dei «mauvais livres», sottoprodotti di un romanticismo degradato, ma anche di aver confuso un rapporto autentico con l'estetica e l'arte con «l'esthétisation de la vie», cioè con la banalizzazione dell'estetica stessa.

6 Portrait(s) d'Emma: d'une femme "écrite» aux femmes décrites di Delphine JаYот passa in rassegna i diversi "ritratti" di Emma Bovary tracciati nel tempo dalla critica letteraria, dividendoli in due periodi: dal 1857 al 1957 e dal 1957 ai nostri giorni. "Romanesque" per Sainte-Beuve, "sensuelle" per Brunetière, rappresentante tipica della piccola borghesia per Lemaître o "déclassée" per Zola, Emma rappresenta nel primo secolo dalla sua comparsa un tipo «identifiable et immuable», mentre, dopo il 1957, la sua immagine diventa più dinamica $\mathrm{e}$, sostenuta dal concetto più generale di bovarysme, evolve verso quell'«universel singulier» che Sartre ha adottato per la lettura di Flaubert e Pierre Michon ha attualizzato per Emma affermando: «Madame Bovary est toutes les femmes».

7 Da un esame analitico del concetto di bovarysme, elaborato da Jules de Gaultier nei due saggi successivi del 1892 e del 1902, Per BUvIK deduce il progressivo spostamento della nozione dall'ambito psicologico a quello filosofico. Enjeux éthiques et esthétiques de la pensée de Jules de Gaultier sur le bovarysme traccia quindi i cambiamenti di un concetto, che da morale e sostanzialmente negativo, diventa, principalmente grazie alla lettura di Nietzsche, un principio estetico, amorale, aperto allo «spectacle prodigieux du monde».

8 La terza sezione, «Enjeux éthiques de l'art», inizia con lo studio di Loïc WINDELS Flaubert, Baudelaire, et la mélancolie. Elemento centrale e abusato della letteratura romantica, la malinconia praticata da Flaubert nei suoi romanzi mostra sia l'uso del cliché che il tentativo di tenerlo consapevolmente a distanza, a differenza di Baudelaire che, pur consapevole del poncif, tende piuttosto a rielaborarlo e a modernizzarlo attraverso «une conscience mélancolique du mal». Anche perché, nella morale baudelairiana, la malinconia tende a confondersi con la noia e con l'accidia e appartiene all'uomo dalla sua origine fino al raggiungimento tragico della lucidità. Il discorso malinconico di Flaubert si iscrive invece per Windels in un'etica che non viene dalla morale, ma dalla 
resistenza alla bêtise e ha quindi a che vedere con l'estetica solo per quel tanto che si sottrae alla configurazione romantica, realizzando «une relève méfiante de la malinconie».

9 In Gustave Flaubert et John Ruskin: l'émergence d'une référence, Déborah BoLTz prende in esame i rapporti fra Flaubert e Ruskin, rapporti certi solo dalla parte di Flaubert, che commenta e annota nel Carnet 13 e nelle note di lettura de L'Esthétique anglaise. Étude sur John Ruskin di Milsand (1864) le teorie artistiche di Ruskin. Secondo l'A. quest'ultima lettura sarebbe anche all'origine della figura del pittore Pellerin nell'Éducation sentimentale.

«Éthique et esthétique», ultima sezione del volume, propone alcuni confronti fra autori, cominciando da Nietzsche contre Flaubert? di Jean-Michel REY, che prende in esame le allusioni fatte dal filosofo tedesco all'opera di Flaubert negli ultimi anni di lavoro. Decisamente critico nei confronti dello scrittore francese, Nietzsche appare soprattutto interessato «à l'étrange force» che traspare dalla scrittura di Flaubert e che gli appare come il frutto di una sorta di odio creativo, della tortura che lo scrittore si autoinfligge in nome della totale devozione all'opera.

11 Flaubert avec Sade, di Pierre-Marc DE BIASI, approfondisce un'affermazione corrente della critica sulla prossimità fra Flaubert e Sade. Fondandosi sulla Correspondance, l'A. rileva un certo numero di allusioni e riferimenti a Sade già dalla fine degli anni $30 \mathrm{e}$ soprattutto la volontà del giovane Flaubert di avere una conoscenza più ampia e diretta dell'opera sadiana, acquisita a partire dal 1840 e già evidente nell'Éducation sentimentale del 1845. Dalla sua accurata disamina, che comprende le accuse di «sadisme» di SainteBeuve a Salammbô, l'A. arriva alla conclusione di una sorta di comunione di intenti fra i due autori, fondata su una «empathie indéfectible» da parte di Flaubert, che è tuttavia lucidamente consapevole dei limiti della sua adesione, segnati fra l'altro dalla totale assenza di auto-ironia dei testi di Sade.

Lo studio successivo riprende lo stesso argomento, dato che Florence PELLEGRINI vi esamina gli Avatars flaubertiens de l'objet sadique: ancora una volta è la Correspondance a fornire il terreno principale di un'indagine sull'utilizzo, da parte di Flaubert, di oggetti e situazioni «sadiques». Il fouet, «essence de l'objet sadien» vi compare in primo piano in tutte le sue «variantes flaubertiennes»-caravache, nerf de bøuf, martinet ecc. -, seguito dalla «violence amoureuse» di Emma Bovary . L'oggetto "sadien» ne esce indubbiamente deformato e spesso edulcorato, ma sempre inquietante nonostante l'involucro saldo dello stile. 